\title{
A Multi-Trait Dynamic User Model for Adaptive Educational Hypermedia Systems (AEHS)
}

\author{
Nafisa A. Hassan \\ Faculty of Computer Science and Information \\ Technology \\ University of West Kordofan \\ Elnihood, Sudan
}

\author{
Noureldien A. Noureldien \\ Faculty of Computer Science and Information \\ Technology \\ University of Science and Technology, \\ Omdurman, Sudan
}

\begin{abstract}
Adaptive educational hypermedia systems (AEHS) is an evolving technology that aims to provide personalized learning content and presentation based on individual user characteristics to fasten and improve self-learning. In the context of AEHS, user characteristics are either static or dynamic. Static users attributes are captured prior using the AEHS, while dynamics ones are captured during using the AEHS. Many dynamic characteristics such as knowledge, learning style, behavior, environment, goals, and preferences have been defined. The user characteristics used in an AEHS are defined by what is known as user model (UM). Today's many AEHS incorporates different static and dynamic user models. In this paper, a user model that incorporates four user dynamic characteristics was proposed. The characteristics engaged are the user's current state of knowledge, learning style, goals, and behavior. The developed user model is assumed to be used in a lesson-based AEHS. A valuation algorithm that based on user navigation links is developed and used to determine the values of the four dynamic characteristics. We claim that this new model is more efficient in adapting the presentation and the learning content that shaping the individual user needs.
\end{abstract}

\section{General Terms}

Adaptive Educational Hypermedia Systems, User Model, Dynamic User Characteristics, Link Analysis Algorithm, and Valuation Algorithm

\section{Keywords}

Adaptive Educational Hypermedia Systems, Dynamic User Model, Dynamic Characteristics, Modeling Techniques.

\section{INTRODUCTION}

Based on hypermedia systems, the Adaptive Educational Hypermedia Systems (AEHS) appeared in the 1990s to tailor personalized learning contents. An AEHS consists of three main components; the Domain Model (DM), the User Model (UM) and the Adaptation Model (AM). The DM defines the subject area and the learning material that has to be used as a learning resource. The $\mathrm{UM}$ is a collection of a user characteristics that the DM content has to be adapted to satisfy them. The AM describes how the content to adapt to satisfy user characteristics defined by the user model [1]. In ref. [2] Saied two major components of the hypermedia can be subject to adaptation, the presentation (content) and the navigation through the hyperspace (links).

User models have been classified either as static or dynamic depending on when updates of the user information take place. In a static user model, user information is collected by queries or by observations and updates takes place only in an initial phase or at regular intervals. In dynamic user model, user information is collected and updated continuously while the user interacts with the system [3].

A Dynamic user model facilitates the implementation of hypermedia applications that adapt themselves at each step to the user such as adaptive educational hypermedia systems (AEHS). AEHS are developed to enhance and improve the learning experiences, user's satisfaction and to maximize the learning quality. Many dynamic user models for AEHS have been proposed, and many systems have been developed to support adaptation. These systems tailored their outputs to meet the individual user's needs.

AEHS is considered by many researchers as an essential approach to improve effectiveness, the efficiency of learning, and increase user's satisfaction [4]. Most of the developed AEHS base their adaptation to user's knowledge [4-8]. Recently, learning styles and user knowledge are combined in some AEHS in refs. [9-14]. Other AEHSs are focus on user's goals [15-17] and user's behavior in [18].

In this paper, a new dynamic user model for lesson-based AEHS was proposed. The model incorporates four dynamic user characteristics; knowledge, learning style, goals, and behavior. In lesson-based AEHS the content is organized into successive lessons. Knowledge tests are used to move from a lesson to the next one. The next lesson content will be adapted based on the characteristics the user reveals in the current lesson session. To evaluate the current user characteristics, the model uses an evaluation algorithm that analyzes navigation links used by the user.

The rest of this paper is organized into five sections: Section 2 provides background on the four user dynamic characteristics implemented by the developed model. Section 3 surveys the related work. In section 4 the proposed user model is presented. Section 5 is devoted to the conclusion and future work.

\section{BACKGROUND}

This section gives a precise definition of the four dynamic characteristics; knowledge, learning styles preference, goals/tasks, and behavior, that have been included in the developed user model, and presents the different modeling techniques that have been used to model these characteristics in current AEHS.

\subsection{Knowledge}

Knowledge is defined as the set of facts, concepts, and skills about a subject or a domain acquired by a user [19]. Many techniques have been used to model knowledge such as 
overlay, stereotype, perturbation, differential, machine learning, and Bayesian networks.

\subsection{Learning Styles Models}

The learning styles characteristics; cognitive, affective and psychological behaviors, can be used as indicators of how learners learn, and interact with the learning material [13, [20, 21]. Learning styles are also defined to refer to individual characteristics and preferred ways of gathering, organizing and thinking about information [22]. Learning styles models have been classified into six models [23]. These models were applicable in AEHS. These models are; Dunn and Dunn, Witkin, Felder and Silverman, Kolb, Honey and Mumford, and Fleming's VARK Model.

\subsection{Goals Models}

The user's goals/tasks are the most changeable user feature that activates adaptation [16]. It is related to the context of a user's work with a hypermedia application rather than with the user as an individual. It informs what the user wants to accomplish by using the application. For example, in information retrieval systems, a user's goal is a search goal; in educational systems is a learning goal; in testing, systems might be a problem-solving one [14, 16] and [24]. In all of these cases, the goal is an answer to the question "What does the user want to achieve?" [24].

It is important to know the goals of the user and to determine the learning strategies. User's goals have many approaches to model and representation in user model such as: A goal catalog approach and plan recognition.

\subsection{Behavior Models}

User behavior influences the way users may or may not want to become more actively involved in their learning process, as well as their interest in preferences for the learning materials. The behavior models include machine learning algorithms, soft computing systems such as; Fuzzy Logic, Neural Networks, Genetic Algorithms, Fuzzy Clustering and NeuroFuzzy systems [25] and the Myer-Briggs Type Indicator (MBTI) model is used in [18].

\section{RELATED WORK}

In knowledge-based AEHSs different knowledge, different models are used. Overlay model is used in representing the student's knowledge in refs. [26-29]. Stereotypes have been used in refs. [4, 5, 29-32].

The differential model has been used in refs. [33, 34]. Perturbation model is used to represents learner knowledge in refs. $[35,36,37]$, and Bayesian models have been used in refs. $[6,7,38,14,39]$.

In learning style-based AEHSs, learning style methods have been used to accommodate the user model to personalization of the learning content. In [40] Dunn and Dunn's model is used. Witkin models are used in [41]. Felder and Silverman's learning styles have been used in refs. [42-45] and [23]. David Kolb's model was used to define the adaptation rules for adapting the learning content and the navigation [46]. The automatic generation of an adapted course page for a student with preferences towards verbal perception modality is used in $[20,11]$. Pioneer AEHSs have implemented Honey and Mumford learning style Model [13] such as; AHA! [28], and INSPIRE [47, 48]. VARK Model is employed to determine student's learning style in refs. [49, 50].
In goals-based AEHSs, a hierarchy of task has been used in refs. $[15,16,17]$ a goal catalog approach also is used. Plan recognition model is used in refs. [14, 27], and finally, data mining techniques used in $[10,17,51]$.

In behavior-based AEHSs, user models are implemented using used machine learning algorithms [52]. In ref. [25] soft computing systems have been used, and Myer Briggs Type Indicator (MBTI) is used in ref. [18].

User models that based on multi-dynamic characteristics are used in many AEHS's. Examples include; knowledge and learning styles $[9,10,11]$, knowledge and goals in refs. [27, $31,53,54]$, knowledge and behavior in ref. [18], knowledge, behavior and learning styles in [26], and knowledge, learning styles and goals in $[55,11]$.

\section{THE PROPOSED USER MODEL}

The proposed dynamic user model is build to support adaptivity in lesson-based AEHS, using knowledge, learning style, goals, and behavior as dynamic user characteristics. In lesson-based AEHS the learning material is designed as a series of in-a-row lessons. The user will log at first to the first lesson. Each lesson is a collection of web pages that present the learning material of that lesson.

The model assumes that the first lesson web pages are designed to be neutral to the three user characteristics; learning style, goals, and behavior. By neutral design, we mean that the content and links are equally support the different sub-characteristics of each the three dynamic characteristics. For example, the number of links presented to support VARK learning style sub-characteristics visual, Auditory, Reading /writing and kinesthetic has to be equal.

For the fourth characteristic, the user knowledge, the user is assumed to pass a knowledge test at the end of each lesson to be eligible to move to the next lesson. The developed model evaluates these four dynamic characteristics for a user and passes the evaluation to the adaptation engine to adapt the next lesson based on the received input.

In the next subsections, we show the user characteristics models that have been implemented, and the valuation algorithm adopted to evaluate these characteristics.

\subsection{Implemented Models}

For knowledge, the model implements overlay and a stereotype model as knowledge are presented as lessons. For learning style preference, the model implements the Fleming's VARK model [22]. For goals, the developed model employ learning and problem-solving goals model, i.e., the goal catalog approach $[14,16]$, where learning goals are relatively stable for a training unit, but the problem- solving goals can shift from one problem to another within the same learning situation.

To determine the user behavior, the model implements the Myer-Briggs Type Indicator model (MBTI) [18]. The model implements only two behaviors, introverts, and extroverts. Introvert define the behavior in which the user is focused on ideas and feelings (inner world), think thoroughly first before acting, while in extravert behavior a user is focused on things and actions (outer world), tend to express emotion freely (act first then reflect).

Fig.1 shows the architecture of the proposed user model. 


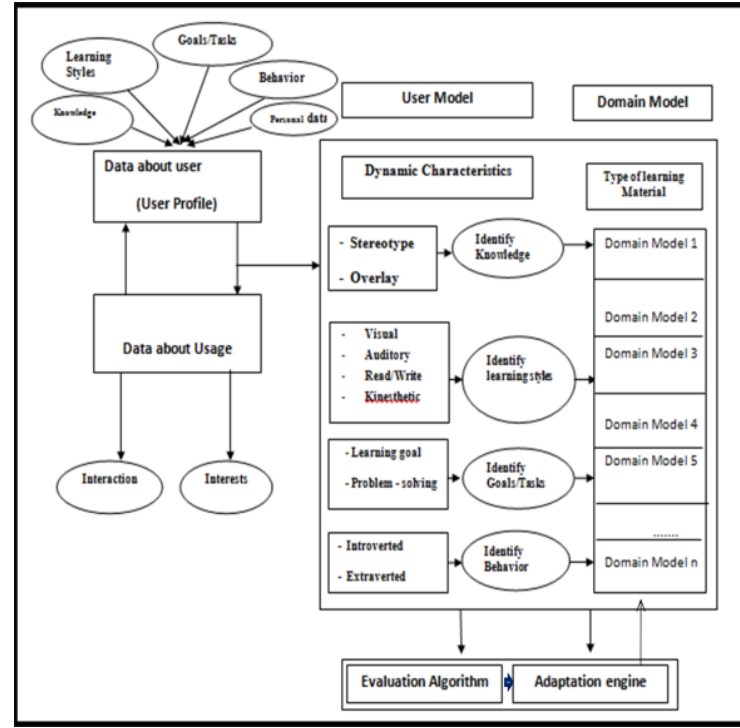

Fig 1: Architecture of the Proposed User Model

The architecture shows that the model is based on four dynamic user characteristics; knowledge, learning styles, goals, and behavior in addition to personal data which is captured during the registration process (i.e., user profile). The valuation logs user navigation links and analyzes these logs to identify user's characteristics possessed during the lesson. The evaluation algorithm passes its valuation to the adaptation engine to generate the lesson.

\subsection{Evaluation Algorithm}

A user characteristics valuation algorithm is developed to evaluate the user's dynamic characteristics before moving to the next lesson. . The valuation algorithm is based on link analysis on the user's set of navigation links in the current lesson before the knowledge test. The algorithm assigns values to user learning style, goals and behavior, and knowledge.

The algorithm is given below

\section{Algorithm}

// to evaluate users learning style, goals, and behavior

1- In the current lesson pages let

$\mathrm{S}=\left\{\mathrm{S}_{1}, \mathrm{~S}_{2}, \mathrm{~S}_{3}, \mathrm{~S}_{4}\right\}$ denotes the set of learning style dedicated links, where $S_{1}, S_{2}, S_{3}$, and $S_{4}$ are subsets of $S$ containing dedicated links to Visual, Aural, Read/write and Kinesthetic respectively.

$\mathrm{G}=\left\{\mathrm{G}_{1}, \mathrm{G}_{2}\right\}$ denotes the set of goals dedicated links, where $\mathrm{G}_{1}$ and $\mathrm{G}_{2}$ are subsets of $\mathrm{G}$ containing dedicated links that support the learning and problem-solving.

$B=\left\{B_{1}, B_{2}\right\}$ denotes the set of behavior dedicated links, where $B_{1}$ and $B_{2}$ are subsets containing dedicated links that reflects introverted and extroverted for users behaviors respectively

2- Let $U=\left\{L_{1}, L_{2}, \ldots, L_{n}\right\}$ denote the set of links navigated by the user before taking the knowledge test.

3- Read $\mathrm{L}_{\mathrm{i}}$, for $1<=\mathrm{i}<=\mathrm{n}$ from $\mathrm{U}$ and determine the set and subset it belongs to, and increment the corresponding counter
4- // to evaluate user knowledge

Read user answers to the knowledge test and calculate his grade $(\mathrm{A} / \mathrm{B} / \mathrm{C} / \mathrm{D} / \mathrm{F})$.

5- // return ratios of user characteristics valuation and grade

Return $\left(S_{1}: S_{2}: S_{3}: S_{4}, G_{1}: G_{2}, B_{1}: B_{2}\right.$, grade)

6- If user grade is F remain in the same lesson else pass the returned values to the adaptation engine.

\subsection{Model Evaluation}

The evaluation of the developed system is a twofold process. Firstly the proposed user model has to be implemented as online AEHS. Secondly, learning metric should be defined and used to compare the learning outcomes achieved by using the implemented system with other AEHS that employ 1, 2 or 3 dynamic characteristics in their user model.

Here we should show how we implement the proposed system. The second part of the evaluation is left as a future work because testing with others AEHS requires acquiring such systems and comprehensive, time exhausting, tests and comparisons.

The user model and domain model of the developed AEHS were implemented using Ajax technology and a MySQL database. The PHP scripting language was used to present a dynamic content and for reading information from web forms and maintaining sessions between web pages. The adaptation model and interface were implemented in HTML environment and enhanced by JavaScript.

Users must be registered to the system. Once a user is registered, a user model will be created to store user information; a unique id is generated for the user for future reference and for tracking the user learning progress. After successful registration, users can $\log$ in to the system. The AEHS system shows the introducing page of lesson 1 (fig. 2) which have been designed regarding content and presentation to support equally the learning styles preferences, the goals, and the behaviour.

The implemented valuation algorithm logs the user's clicks on links and identifies their types and deduces user dynamic characteristic values.

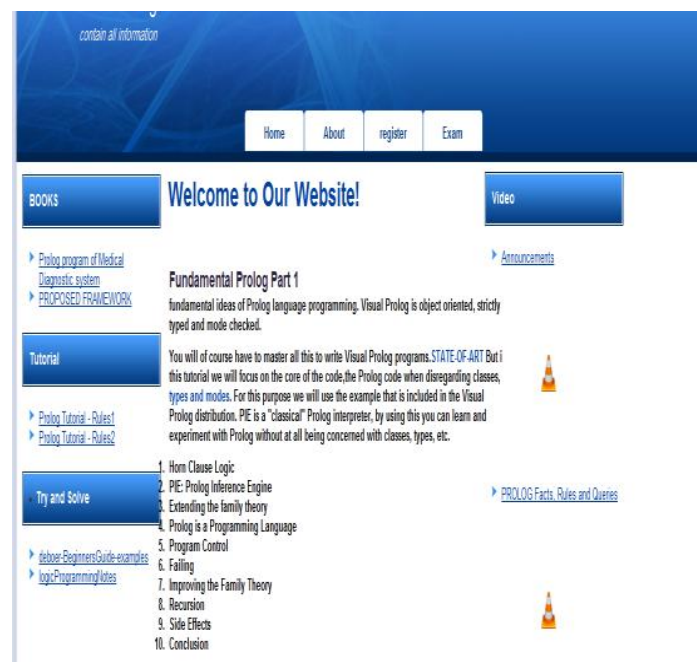

Fig 2: Lessons 1: Support equally user characteristics 
Every lesson starts with objectives, followed by a small introduction, then the lesson content with concrete examples, and finally and a knowledge test is presented to move to the next lesson. If the user passes the test, the system will allow him to move to the next concepts using the next lesson. On the other hand, if he fails, the system will ask him to repeat the lesson and offers the user the option to guide him forward.

On moving to the next lesson, the valuation algorithm will determine the ratios of user's dynamic characteristics; learning styles, goals, and behavior and passes these ratios with the knowledge test grade to the adaptation module. Fig. 3 shows lesson two whose content and presentation is adapted to the passed values.

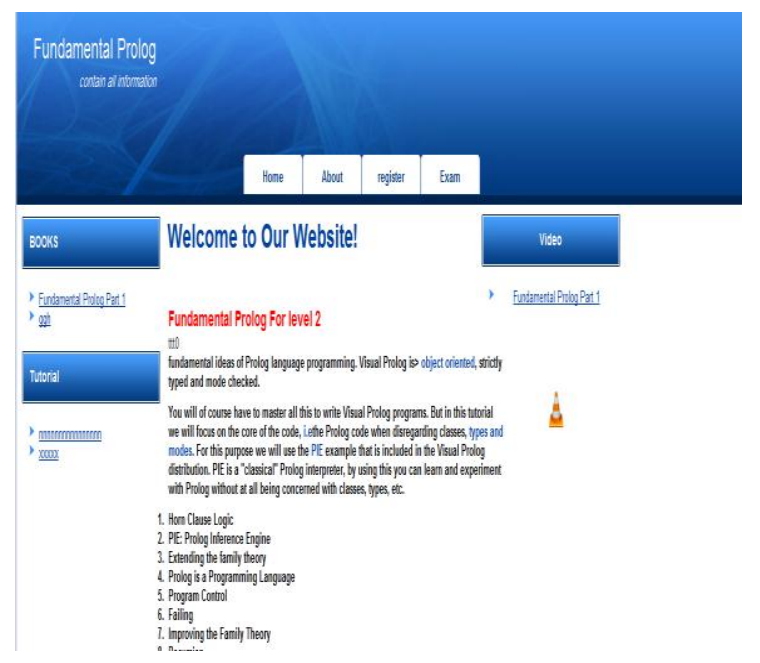

Fig3: Navigation and Content Adaptation

\section{CONCLUSION}

This paper presents a new dynamic user model for a lesson based-AEHS. The model incorporates four dynamic user characteristics; knowledge, learning style, goals, and behavior. To move to the next lesson, knowledge tests are used, and the next lesson content will be adapted based on the characteristics the user revealed in the current lesson session and the grade obtained in the knowledge test. To evaluate the current user characteristics the model uses evaluation algorithms that analyze the user navigation links.

\section{REFERENCES}

[1] DeBra, P., and Calvi, L. 1998. AHA! An Open Adaptive Hypermedia Architecture. New Review of Hypermedia and Multimedia 4 (1), pp.115-139.

[2] Brusilovsky, P. 2001. Adaptive hypermedia. User Modeling, and User-Adapted Interaction. 11 (1/2), pp. $87-110$.

[3] Baldiris, S., Graf, S., and Fabregat, R. 2011. Dynamic User Modeling and Adaptation based on Learning Styles for Supporting Semi-Automatic Generation of IMS Learning Design. $11^{\text {th }}$ IEEE International Conference on Advanced Learning Technologies. pp. 218-220.

[4] Colak, I., Sagiroglu, S., and Tolga, H. 2008. A User Modeling Approach to Web Based Adaptive Educational Hypermedia Systems. Seventh International Conference on Machine Learning and Applications, ICMLA '08.

[5] Brusilovsky, P. 1996. Methods and techniques of adaptive hypermedia, Special Issue on Adaptive
Hypertext and Hypermedia. User Modeling and UserAdapted Interaction, vol. 6, pp. 87- 129.

[6] Esichaikul, V. Lamnoi, S., and Bechter, C. 2011. Student Modeling in Adaptive E-Learning systems. Field Dependent and Field-Independent Cognitive Styles and Their Educational Implications. Review of Educational Research, 47 (1), 1-64.

[7] Homsi, M., Lutfi, R., Maria, R., and Barakat, G. 2008. Student Modeling Using NN-HMM for EFL Course. In Proc. of the international conference on Information and Communication Technologies: From Theory to Applications, ICTTA.

[8] Stash, N., and De Bra. P., 2004. Incorporating Cognitive Styles in AHA! (The Adaptive Hypermedia Architecture). Proceeding of the IASTED International Conference Web-Based Education, Innsbruck, Austria, PP. 378-383.

[9] De Koch, N.P. 2001. Software Engineering for Adaptive Hypermedia Systems Reference Model. Ph.D. Thesis, University Munchen.

[10] Bayasut, B., Pramudya, G., and Basiron, H. 2013. ULUL-ILM the Design of Web-Based Adaptive Educational Hypermedia System Based on Learning Style. 3th International Conference on Intelligent Systems Design and Applications (ISDA), PP.147 - 152.

[11] Popescu, E., and Badica, C. 2011. Creating a Personalized Artificial Intelligence Course: WELSA case study. International Journal of Information Systems and Social Change (IJISSC), vol. 2, no. 1, pp. 31 -47, IEEE.

[12] Popescu, E. 2008. Dynamic adaptive hypermedia systems for e-learning. Ph.D. thesis, EUniversite de Technologie de Compiegne.

[13] Mustafa, Y.E.A., and Sharif, S.M. 2011. An approach to adaptive e-learning hypermedia system based on learning styles (AEHS-LS): Implementation and evaluation. International Journal of Library and Information Science, 3(1), pp.15-28.

[14] Nguyen, L. 2014. A User Modeling System for Adaptive Learning. Available at: <http://www.researchgate.net/publication/282691564> [Accessed 19 Dec. 2015].

[15] Brusilovsky, P., and Millan, E. 2007. User models for adaptive hypermedia and adaptive educational systems. In the adaptive web: Springer-Verlag.

[16] Vassileva, J. 1996. A task-centered approach for user modeling in a hypermedia-based information system. In Proceedings of the 4th International Conference on User Modeling, Hyannis, MA, pp. 115-120.

[17] [17] Brusilovsky, P., and Cooper, D.W. 2002. Domain, Task, and User Models for an Adaptive Hypermedia Performance Support System. In: Y., Gil, and Leake, D.B., eds., Proc. of 2002. International Conference on Intelligent User Interfaces, ACM Press, pp. 23-30.

[18] Al-Dujaily, A. 2007. Personality effect in the design of adaptive e-learning systems, Ph.D.Thesis. Massey University, Auckland.

[19] Tadlaoui, M.A., et al. 2016. Learner Modeling in Adaptive Educational Systems: A Comparative Study. 
International Journal of Modern Education and Computer Science, 8(3): pp.1.

[20] Samia, D., and Abdelkrim, A. 2012. An Adaptive Educational Hypermedia System Integrating Learning Styles: Model and Experiment. International Conference on Education and eLearning Innovations, IEEE.

[21] Mulwa, C., et al. 2010. Adaptive educational hypermedia systems in technology enhanced learning: a literature review. In Proceedings of the 2010 ACM conference on Information technology education: ACM.

[22] Fleming, D.N. 2012. Teaching and Learning Styles: VARK Strategies. ISBN Number: ISBN 978-0-47307956-7. Available at: 〈http:// www.vark-learn.com>.

[23] Coffield, F. Moseley, D. Hall, E., and Ecclestone, K. 2004. Learning Styles and Pedagogy in Post- Learning. A Systematic and Critical Review, Learning and Skills Research Centre, UK.

[24] Encarnação, L.M.1997. Multi-level user support through adaptive hypermedia: A high application independent help component. "Proc. of 1997 International Conference on Intelligent User Interfaces, pp.187-194 ACM.

[25] Martinez, E.F., et al. 2005. Modeling Human Behavior in User-Adaptive Systems: Recent Advances Using Soft Computing Techniques. Expert System with pplications, 29 (2).

[26] Momar, V. H-Bozic, N., and Zokovic, D.P. (2003). Approaches to Online Testing in Web-based Educational Systems, IEEE, EUROCON Ljubljana, Slovenia, pp.343346.

[27] Brusilovsky, P., and Rizzo, R. 2002. Map-Based Horizontal Navigation in Educational Hypertext. Proceedings of the thirteenth ACM conference, 2002 dl.acm.org

[28] De Bra, P, et al. 2003. AHA! The Adaptive Hypermedia Architecture. dl.acm.org

[29] Brusilovsky, P., and Pesin, L. 1994a-1994b. Adaptive Navigation Support in Educational Hypermedia: An Evaluation of The ISIS - Tutor.

[30] Surjono, H.D., and Maltby, J.R. 2003. Adaptive Educational Hypermedia-Based on Multiple Student Characteristics. In, ICWL: Springer.

[31] Colan, O., Dagger, D., and Wade, V. 2002. Towards a Standards-based Approach to e-Learning Personalization using Reusable Learning Objects. In world conference on e-Learning in Corp., Govt., Health and Higher Ed. AACE, pp.210-217.

[32] Maier, P., Armstrong, R., Hall, W., and Hong, M. 2005. An Adaptive Web-based Learning Application. Learning, Media and Technology, volume 30, issue 3, pp. 281-297.

[33] Martinez, E.F., et al. 2005. Modeling Human Behavior in User-Adaptive Systems: Recent Advances Using Soft Computing Techniques. Expert System with pplications, 29 (2).

[34] Sleeman, D. (1984). Inferring student models for intelligent computer-aided instruction. In: Machine Learning, an Artificial Intelligence Approach. SpringerVerlag, Berlin.
[35] Kay, J. 2000. User Modeling for Adaptation. In Stephanidis, C., ed., User Interfaces for All: Concepts, Methods, and Tools. Florence, pp. 271-294

[36] Nguyen, L., and Do, P. 2008. Learner Model in Adaptive Learning. In World Academy of Science, Engineering, and Technology, pp.396-401

[37] Brown, J. S., and Burton, R. 1978. A paradigmatic example of an artificially intelligent instructional system. International Journal of Man-Machine Studies, 10, pp.323-339.

[38] Stash, N., and De Bra. P., 2004. Incorporating Cognitive Styles in AHA! (The Adaptive Hypermedia Architecture). Proceeding of the IASTED International Conference Web-Based Education, Innsbruck, Austria, PP. 378-383.

[39] Laroussi, M. 2008. Conception et Realization d'un Système Didactique Hypermedia Adaptive: CAMELEON. Ph.D. Thesis, retrieved January 15 , Available <http://wwwis.win.tue.nl/ah/thesis/laroussi.pdf >.

[40] Wolf, C. 2003. iWeaver: Towards learning style-based eLearning in Computer Science Education. Australasian Computing Education Conference (ACE2003), Research and Practice in Information Technology, Vol.20.

[41] Witkin, H.A., Moore, C.A. Goodenough. D. R., and Cox, P. W. 1977. Field-Dependent and FieldIndependent. Cognitive Styles and Their Educational Implications, Review of Educational Research Vol. 47, No. 1, pp. 1-64.

[42] Franzoni, A.L., and Assar, S. 2009. Student Learning Styles Method Based On Teaching Strategies and Electronic Media. Educational Technology \& Society, 12(4), pp.15-29.

[43] Bunt, A., and Conati, C. 2003. Probabilistic Student Modeling to Improve Exploratory Behavior. In User Modeling and User-Adapted Interaction, 13(3).

[44] Karagiannis, I., and M. Satratzemi, M. 2014. Comparing LMS and AEHS: challenges for Improvement with Exploitation of Data Mining. IEEE 14th International Conference on Advanced Learning Technologies, pp. 6566, IEEE.

[45] Adetunji A., and Ademola A. 2014. A Proposed Architectural Model for an Automatic Adaptive eLearning System Based on Users Learning Style. International Journal of Advanced Computer Science and Applications (IJACSA), 5(4).

[46] Bajraktarevic N., Hall, W., and Fullick, P. 2003. ILASH: Incorporating Learning Strategies in Hypermedia. In Fourteenth Conference on Hypertext and Hypermedia.

[47] Georgiou, D. A, Botsios, S., and Mitropoulou, V. Papaioannou, M. Schizas, C. and Tsoulouhas, G. 2011. Learning Style Recognition Based on an Adjustable Three- Layer Fuzzy Cognitive Map. JAISCR, Vol.1, No.4, pp.333-347.

[48] Grigoriadou, M., Papanikolaou, K., Kornilakis, H., and Magoulas, G. 2001. INSPIRE, An Intelligent System for Personalized Instruction in a Remote Environment. In the 3rd Workshop on Adaptive Hypertext and Hypermedia, Sonthofen, Germany. 
[49] Stash, N., Cristea, A., and De Bra, P., 2004. Authoring of Learning Styles in Adaptive Hypermedia: Problems and Solutions. ACM. New York, New York, USA

[50] Soudani, L. S., and Aghaee, N.G. 2013. A Model for Personalized Intelligent Tutoring Systems. International Journal of Advanced Computer Technology (IJACT) 2(5), pp. $9-13$.

[51] Martinez, E.F., et al. 2006. Survey of Data Mining Approach to User Modeling for Adaptive Hypermedia. IEEE Transaction on Systems, 36 (6).

[52] Han J., and Michelime 2006. Data Mining Concept and Techniques. Second Edition, by Elsevier Inc, San Francisco. CA 94111.
[53] DeBra, P., and Calvi, L. 1998. AHA! An Open Adaptive Hypermedia Architecture. New Review of Hypermedia and Multimedia 4 (1), pp.115-139.

[54] Brusilovsky, P. and Pesin, L., 1998. Adaptive Navigation Support in Educational Hypermedia. An Evaluation of the ISIS-Tutor, pp.1- 23

[55] Brusilovsky, P. 1992. A framework for intelligent knowledge sequencing and task sequencing. In: Proc. of Second International Conference on Intelligent Tutoring Systems, ITS'92. Springer-Verlag, 499-506. 